\title{
Basic research, experimental surgery and clinical research: where there is science, there is better treatment
}

\author{
G. Campanelli ${ }^{1}$
}

Published online: 17 July 2020

C) Springer-Verlag France SAS, part of Springer Nature 2020

Conducting research in the field of surgery can be a real challenge, for several reasons. First, the chances of accessing funds and using top-level laboratories are limited; second, experimental surgery is becoming increasingly difficult, both ethically and financially; and third, clinical research generally faces numerous hurdles (organisational and in terms of the necessary authorisations) that make the whole process difficult and laborious. We need to only think of the growing difficulties, not least financial, faced by those wanting to carry out level I trials.

And yet the scientific method is based, essentially, on "freedom" of thought and action, although we have to remember that scientific output can be used to advance medicine and for the good of humankind, humans cannot be used to test treatments and advance scientific research. The scientific method is also based on the comparison of techniques, on peer exchanges, and on objective and real-life evaluation of results.

This latter point is particularly crucial, raising two issues that emerge as particular weaknesses of this system.

The first is the problem of attachment to certain specific techniques and surgical procedures, which can make it difficult to abandon established techniques, or even compare them with others, and can somehow produce "distorted" judgments, leading surgeons to harbour, a priori, a preference for the standard technique.

The second problem is that it is often objectively difficult, and sometimes even impossible, to verify surgical results in the medium and long term.

Take the case of very common and widespread surgeries, like hernia and abdominal wall repair. In more advanced centres, it is now practically "obligatory" to keep patient registries, and this makes post-operative follow-up a whole lot

G. Campanelli

hernia.editorialoffice@gmail.com

1 University of Insubria, Gruppo Ospedaliero San Donato, Milan, Italy easier. But the problem is, these are procedures that are very widely performed in hospitals of all levels, across all five continents of the world. Clearly, then, the chances of achieving long-term follow-up of the vast quantity of patients who undergo them are slim to say the least.

All this leads to poor reporting and to under reporting, and, therefore, ultimately to considerable difficulties conducting scientific comparisons of techniques, approaches and devices that have credibility, and can, therefore, offer a high level of evidence.

And if we also consider that the methodology behind the production of original papers, reviews, guidelines, and so on, requires consultation and consideration of high levels of evidence, then the difficulties facing those seeking to produce quality papers are clear to see.

So, what is the answer?

It lies in the creation, laborious and difficult but absolutely necessary, of registries validated by external reviewers, because these databases overcome the huge problem of the absence of long-term monitoring of results, and, therefore, provide the credibility that is sorely needed.

This issue of Hernia takes a look, among other things, at the whole follow-up problem, having asked internationally renowned surgeons (who have created or contributed to registry databases) to respond to a letter we received about this very difficult problem for our particular branch of surgery.

I look forward to the constructive discussion that will undoubtedly be generated as a result of our having highlighted this important issue concerning the scientific side of our surgical activity.

I wish to close by reminding you that in an upcoming issue (before the end of the year), we will be focusing extensively on another aspect of scientific research in our sector, through a special forum on experimental surgery and bioengineering. 
Publisher's Note Springer Nature remains neutral with regard to jurisdictional claims in published maps and institutional affiliations. 